\title{
ON AN ANGLE BETWEEN TWO RINGS
}

\section{Grzegorz RzadKowskI}

Jakóbczak and Mazur found the $L^{2}$-angle between two concentric rings on the complex plane. In this note we investigate the same case but for spaces of square integrable functions with various weights. Moreover the continuity of the $L^{2}$-angle for the Fock space is examined.

\section{INTRODUCTION}

The alternating projections and $L^{2}$-angle in the theory of the Bergman function were introduced by Ramadanov and Skwarczyński [2] and Skwarczyński [3, 4]. Jakóbczak and Mazur [1] and Skwarczyński $[5,6]$ have calculated the $L^{2}$-angle for many pairs of domains.

Let $A$ and $B$ be two domains in $C$ with nonempty intersection and put $D=A \cup B$. Let a function $\sigma(z)$, positive and continuous in $D$, be a weight-function. By $L^{2}(D, \sigma)$ we denote the class of all complex valued functions $f$ defined in $D$ with the norm $\|f\|=\left(\int_{D} f(z) \cdot \overline{f(z)} \sigma(z) d m(z)\right)^{1 / 2}<\infty$ (where $m$ stands for the Lebesgue measure in C) and scalar product $\langle f, g\rangle=\int_{D} f(z) \overline{g(z)} \sigma(z) d m(z)$.

Set $F=\dot{L}^{2} H(D, \sigma):=\left\{f \in L^{2}(D, \sigma): f\right.$ is holomorphic in $\left.D\right\}$ and $F_{i}, i=1,2$, the subspaces of $L^{2}(D, \sigma)$ consisting of functions holomorphic in $A$ and $B$ respectively. The $L^{2}$-angle $\gamma(A, B) \in[0, \pi / 2]$ between $A$ and $B$ is given by:

$$
\cos \gamma(A, B)=\sup \left\{\frac{\left|\left\langle f_{1}, f_{2}\right\rangle\right|}{\left\|f_{1}\right\| \cdot\left\|f_{2}\right\|}: f_{i} \in F_{i} \backslash\{0\}, f_{i} \perp F, i=1,2\right\} \text {. }
$$

Under the additional assumption that $L^{2} H(A, \sigma) \neq 0$ or $L^{2} H(B, \sigma) \neq 0$ the same argument as in the case of the uniform weight $(\sigma \equiv 1)$ (see $[2,6])$ leads to:

$$
\cos ^{2} \gamma(A, B)=\sup \left\{\frac{\|f\|_{A \backslash B}^{2}+\|\hat{f}\|_{B}^{2}}{\|f\|_{A}^{2}+\|f\|_{B \backslash A}^{2}}: f \in F_{1} \backslash\{0\}, f \perp F,\right.
$$

$$
f \text { holomorphic in } \operatorname{In}\{(B \backslash A)\}
$$

where $\widehat{f}$ is the Bergman projection of $\left.f\right|_{B}$ in $B$. We shall need the following two easy lemmas subsequently.

Received 14 January 1990

I thank Professor $M$. Skwarczyński for suggesting the problem and for numerous valuable discussions and advice during the preparation of the paper.

Copyright Clearance Centre, Inc. Serial-fee code: 0004-9729/91 \$A2.00+0.00. 
Lemma 1. If the functions $f(x), g(x), h(x)$ defined in $[a, b] \subset \mathbf{R}$ are positive and continuous and moreover $f$ and $g / h$ are both strictly increasing (or both strictly decreasing) then:

$$
\frac{\int_{a}^{b} f(x) \cdot g(x) d x}{\int_{a}^{b} g(x) d x}>\frac{\int_{a}^{b} f(x) \cdot h(x) d x}{\int_{a}^{b} h(x) d x}
$$

Proof: The inequality (3) is equivalent to

$$
\int_{a}^{b} f(x) \cdot g(x) d x \cdot \int_{a}^{b} h(y) d y-\int_{a}^{b} f(x) \cdot h(x) d x \int_{a}^{b} g(y) d y>0
$$

We have

$$
\begin{array}{rl}
\int_{a}^{b} & f(x) \cdot g(x) d x \cdot \int_{a}^{b} h(y) d y-\int_{a}^{b} f(x) \cdot h(x) d x \cdot \int_{a}^{b} g(y) d y \\
& =\int_{a}^{b} \int_{a}^{b} f(x)(g(x) \cdot h(y)-h(x) \cdot g(y)) d y d x \\
& =\int_{a}^{b} \int_{a}^{x}(f(x)-f(y)) \cdot(g(x) \cdot h(y)-h(x) \cdot g(y)) d y d x>0 .
\end{array}
$$

This ends the proof.

Lemma 2. If the function $f(x)$ is continuous and positive in $\mathrm{R}$ and $a<b<c<$ $d$, then the function

$$
\frac{\int_{c}^{d} f(x) d x}{\int_{b}^{d} f(x) d x}
$$

is strictly increasing with respect to $d$, and the function

$$
\frac{\int_{a}^{b} f(x) d x}{\int_{a}^{c} f(x) d x}
$$

is strictly decreasing with respect to $a$.

Proof: The lemma follows immediately by differentiation of (4) and (5) with respect to $d$ and $a$ respectively. We omit an easy calculation. 


\section{A CLASS OF THE WEIGHT-FUNCTIONS}

For our purposes it is very useful to define a special class of the weight-functions. In this paper we investigate only such weights $\sigma(z)$ for which there exists a function $w(x), x>0$, with $\sigma(z)=w\left(|z|^{2}\right)$ and for which the following conditions are fulfilled:

$$
\begin{aligned}
& \text { (1) } w(x)>0 \\
& \text { (2) } w \in C^{1}\left(R_{+}\right) \\
& \text {(3) the function } \frac{x \cdot w^{\prime}(x)}{w(x)} \text { is non-increasing. }
\end{aligned}
$$

Examples of such functions are: the positive constant function, $x^{k}$ (for any $k \in \mathbf{R}$ ), $\exp (-x)$. In the last case the space $L^{2} H\left(\mathrm{C}, \exp \left(-|z|^{2}\right)\right)$ is called the Fock space.

\section{RINGS AND THE $L^{2}$-ANGLE}

Let $A=\left\{z \in \mathrm{C}: 0<r_{1}<|z|<r_{2}\right\}, B=\left\{z \in \mathrm{C}: R_{1}<|z|<R_{2}\right\}$ where $r_{1}<R_{1}<r_{2}<R_{2}$.

We take as the weight a function $\sigma(z)=w\left(|z|^{2}\right)$ which fulfills all conditions $\left({ }^{*}\right)$. The same argument as that carried out by Jakóbczak and Mazur [1] (in the case of uniform weight) leads to

$$
\cos ^{2} \gamma(A, B)=\sup _{n \in Z} W(n) \text { where } W(n)=\frac{\left\|z^{n}\right\|_{A \backslash B}^{2} \cdot\left\|z^{n}\right\|_{B \backslash A}^{2}}{\left\|z^{n}\right\|_{A}^{2} \cdot\left\|z^{n}\right\|_{B}^{2}} .
$$

Substituting in (6): $x=|z|^{2}, a=r_{1}^{2}, b=R_{1}^{2}, c=r_{2}^{2}, d=R_{2}^{2}$, we obtain

$$
W(n)=\frac{\int_{a}^{b} w(x) x^{n} d x \int_{c}^{d} w(x) x^{n} d x}{\int_{a}^{c} w(x) x^{n} d x \int_{b}^{d} w(x) x^{n} d x} .
$$

Assuming that $n \in \mathbf{R}$, we expand (7) to a real function defined in $\mathbf{R}$.

Jakóbczak and Mazur [1] proved that in case of the uniform weight (that is, $w \equiv 1$ ), the expression $W(n)$ attains its maximum, in respect of $n \in Z$, at the point $n_{0}=-1$, and in this case the $L^{2}$-angle between the rings $A$ and $B$ was explicitly calculated.

We shall prove now

ThEOREM 1. If a function $w(x)$ satisfies $\left({ }^{*}\right)$ then the function $W(n) n \in R$ has a single local maximum. It is simultaneously the supremum of $W(n)$ in $R$.

Proof: The first derivative with respect to $n$ of $W(n)$ is

$$
W^{\prime}(n)=W(n) \cdot\left(G_{a b}(n)-G_{a c}(n)+G_{c d}(n)-G_{b d}(n)\right)
$$


where we have denoted

$$
G_{\alpha \beta}(n):=\frac{\int_{\alpha}^{\beta} w(x) \cdot x^{n} \cdot \ln x d x}{\int_{\alpha}^{\beta} w(x) \cdot x^{n} d x} \quad 0<\alpha<\beta .
$$

Notice that the function $G_{\alpha \beta}(n)$ is strictly increasing in respect of $n$ in view of

$$
\begin{aligned}
G_{\alpha \beta}^{\prime}(n) & =\frac{\int_{\alpha}^{\beta} w(x) x^{n} \ln ^{2} x d x \cdot \int_{\alpha}^{\beta} w(x) x^{n} d x-\left(\int_{\alpha}^{\beta} w(x) x^{n} \ln x d x\right)^{2}}{\left(\int_{\alpha}^{\beta} w(x) x^{n} d x\right)^{2}} \\
& =\frac{\int_{\alpha}^{\beta} \int_{\alpha}^{\beta} w(x) w(y) x^{n} y^{n} \ln x \cdot \ln (x / y) d x d y}{\int_{\alpha}^{\beta} \int_{\alpha}^{\beta} w(x) w(y) x^{n} y^{n} d x d y} \\
& =\frac{\int_{\alpha}^{\beta} \int_{\alpha}^{x} w(x) w(y) x^{n} y^{n}(\ln (x / y))^{2} d y d x}{2 \int_{\alpha}^{\beta} \int_{\alpha}^{x} w(x) w(y) x^{n} y^{n} d y d x}>0 .
\end{aligned}
$$

We shall prove now that

$$
G_{a b}^{\prime}(n)<G_{a c}^{\prime}(n)
$$

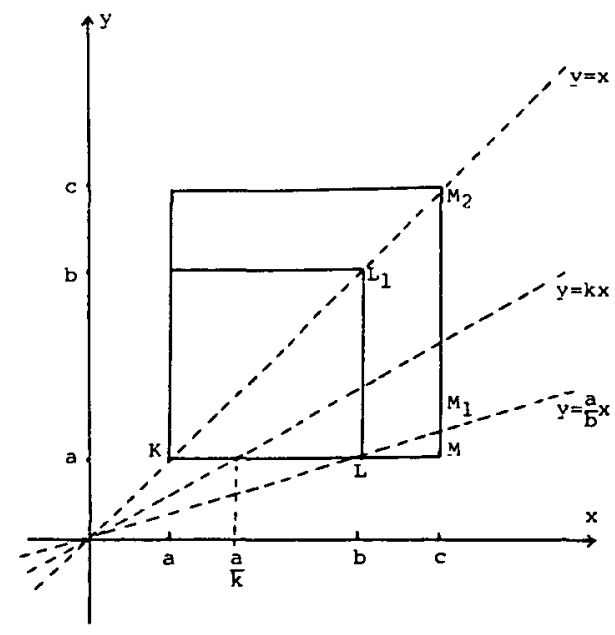

Figure 1

Denote the triangles $K M M_{2}, L M M_{1}, K L L_{1}$ by $P, S, T$ respectively and the quadrilateral $K L M_{1} M_{2}$ by $Q$ (see Figure 1 ).

We have

$$
G_{a b}^{\prime}(n)=\frac{\iint_{T} w(x) w(y) x^{n} y^{n} \cdot(\ln (x / y))^{2} d x d y}{2 \iint_{T} w(x) w(y) x^{n} y^{n} d x d y}
$$


and

$$
G_{a c}^{\prime}(n)=\frac{\iint_{P} w(x) w(y) x^{n} y^{n} \cdot(\ln (x / y))^{2} d x d y}{2 \iint_{P} w(x) w(y) x^{n} y^{n} d x d y}=\frac{\iint_{Q}+\iint_{S}}{2 \iint_{Q}+2 \iint_{S}}
$$

First we show that

$$
\frac{\iint_{Q} w(x) w(y) x^{n} y^{n} \cdot(\ln (x / y))^{2} d x d y}{2 \iint_{Q} w(x) w(y) x^{n} y^{n} d x d y}>G_{a b}^{\prime}(n) .
$$

(9) follows from (10) since in the triangle $S$ the function $\ln ^{2}(x / y)$ takes bigger values than in the quadrilateral $Q$. Each of the four integrals in (10) we write in polar coordinates $(r, \varphi), x=r \cos \varphi, y=r \sin \varphi ; \varphi \in[\arctan a / b, \pi / 4]$, and the integration with respect to $r$ we replace by integration with respect to $x$. Thus $\left(k:=\tan \varphi, \varphi_{0}:=\arctan a / b\right)$;

$$
\begin{aligned}
\iint_{T} w(x) & w(y) x^{n} y^{n} d x d y \\
= & \int_{\varphi_{0}}^{\pi / 4} d \varphi \int_{a / k}^{b} w(x) w(k x) x^{n}(k x)^{n} \sqrt{x^{2}+(k x)^{2}} \sqrt{1+k^{2}} d x \\
= & \int_{\varphi_{0}}^{\pi / 4} d \varphi k^{n}\left(1+k^{2}\right) \int_{a / k}^{b} w(x) w(k x) x^{2 n+1} d x
\end{aligned}
$$

and similarly

$$
\begin{aligned}
\iint_{Q} w(x) w(y) x^{n} y^{n} d x d y \\
\quad=\int_{\varphi_{0}}^{\pi / 4} d \varphi k^{n}\left(1+k^{2}\right) \int_{a / k}^{c} w(x) w(k x) x^{2 n+1} d x
\end{aligned}
$$

Two integrands of the numerators of (10) differ from the integrands of (11) and (12) only in the factor $\ln ^{2} k$. Also $\ln ^{2} k$ is decreasing with respect to $k$ (and $\varphi$ ), and by Lemma 1 , to show (10) it suffices to prove that the expression

$$
\frac{\int_{a / k}^{c} w(x) w(k x) x^{2 n+1} d x}{\int_{a / k}^{b} w(x) w(k x) x^{2 n+1} d x}
$$

is decreasing with respect to $k$ (and therefore $\varphi$ ). 
The numerator of the derivative of (13) with respect to $k$ is:

$$
\begin{aligned}
& \left(\frac{a}{k^{2}} \cdot w\left(\frac{a}{k}\right) \cdot w(a)\left(\frac{a}{k}\right)^{2 n+1}+\int_{a / k}^{c} w(x) w^{\prime}(k x) x^{2 n+2} d x\right) \cdot \int_{a / k}^{b} w(x) w(k x) x^{2 n+1} d x \\
& -\left(\frac{a}{k^{2}} \cdot w\left(\frac{a}{k}\right) \cdot w(a)\left(\frac{a}{k}\right)^{2 n+1}+\int_{a / k}^{b} w(x) w^{\prime}(k x) x^{2 n+2} d x\right) \cdot \int_{a / k}^{c} w(x) w(k x) x^{2 n+1} d x \\
& =-\frac{1}{k} w\left(\frac{a}{k}\right) w(a)\left(\frac{a}{k}\right)^{2 n+2} \cdot \int_{b}^{c} w(x) w(k x) x^{2 n+1} d x \\
& +\int_{a / k}^{c} w(x) w^{\prime}(k x) x^{2 n+2} d x \cdot \int_{a / k}^{b} w(x) w(k x) x^{2 n+1} d x \\
& -\int_{a / k}^{b} w(x) w^{\prime}(k x) x^{2 n+2} d x \cdot \int_{a / k}^{c} w(x) w(k x) x^{2 n+1} d x \\
& =-\frac{1}{k} w\left(\frac{a}{k}\right) w(a)\left(\frac{a}{k}\right)^{2 n+2} \int_{b}^{c} w(x) w(k x) x^{2 n+1} d x \\
& +\int_{b}^{c} w(x) w^{\prime}(k x) x^{2 n+2} d x \cdot \int_{a / k}^{b} w(x) w(k x) x^{2 n+1} d x \\
& \quad-\int_{a / k}^{b} w(x) w^{\prime}(k x) x^{2 n+2} d x \cdot \int_{b}^{c} w(x) w(k x) x^{2 n+1} d x \\
& =-\frac{1}{k} w\left(\frac{a}{k}\right) w(a)\left(\frac{a}{k}\right)^{2 n+2} \int_{b}^{c} w(x) w(k x) x^{2 n+1} d x \\
& +\int_{b}^{c} \int_{a / k}^{b} w(x) w(y)(x y)^{2 n+1}\left(w(k x) w^{\prime}(k y) y-w^{\prime}(k x) w(k y) x\right) d x d y<0
\end{aligned}
$$

The second component of the above expression is nonpositive because in the rectangle $(a / k \leqslant x \leqslant b) \times(b \leqslant y \leqslant c)$ the conditions $\left({ }^{*}\right)$ give

$$
w(k x) \cdot w^{\prime}(k y) y-w^{\prime}(k x) \cdot w(k y) x \leqslant 0
$$

Thus we have proved (10) and therefore (9).

By the same manner one can prove that

$$
G_{c d}^{\prime}(n)<G_{b d}^{\prime}(n)
$$

Finally in the proof of (14) it remains to observe that the expression

$$
\frac{\int_{b / k}^{d} w(x) \cdot w(k x) \cdot x^{2 n+1} d x}{\int_{c / k}^{d} w(x) w(k x) x^{2 n+1} d x}
$$


is decreasing with respect to $k$.

The numerator of the derivative, with respect to $k$, of (15), after similar calculation as was carried out for (13), is

$$
\begin{aligned}
& -\frac{1}{k} w(d) \cdot w(k d) d^{2 n+2} \int_{b / k}^{c / k} w(x) w(k x) x^{2 n+1} d x \\
& +\frac{1}{k} \int_{b / k}^{c / k} \int_{c / k}^{d} w(k x) w(k y)(x y)^{2 n+1}\left(w^{\prime}(x) \cdot w(y) \cdot x-w(x) w^{\prime}(y) y\right) d x d y \\
& \quad<0 .
\end{aligned}
$$

The second component of the above sum is nonpositive since in the rectangle $(c / k \leqslant x \leqslant d$ $(b / k \leqslant y \leqslant c / k)$ the conditions $\left({ }^{*}\right)$ give

$$
w^{\prime}(x) \cdot w(y) \cdot x-w(x) \cdot w^{\prime}(y) \cdot y \leqslant 0 .
$$

By (9) and (14) it follows that the expression in brackets of (8) is strictly decreasing with respect to $n$.

Moreover it is easy to verify that

$$
\lim _{n \rightarrow-\infty} G_{\alpha \beta}(n)=\lim _{n \rightarrow-\infty} \frac{\int_{\alpha}^{\beta} w(x) x^{n} \ln x d x}{\int_{\alpha}^{\beta} w(x) x^{n} d x}=\ln \alpha
$$

and analogously

$$
\lim _{n \rightarrow \infty} G_{\alpha \beta}(n)=\ln \beta .
$$

Therefore by (16) and (17) we have

$$
\lim _{n \rightarrow-\infty} \frac{W^{\prime}(n)}{W(n)}=\ln c-\ln b>0
$$

and

$$
\lim _{n \rightarrow \infty} \frac{W^{\prime}(n)}{W(n)}=\ln b-\ln c<0 .
$$

Thus by (18) and (19) the derivative $W^{\prime}(n)$ has exactly one zero, say $n_{0} \in \mathbf{R}$, at which $W(n)$ attains the global maximum. This ends the proof of Theorem 1.

REMARK 1. Our proper problem is to find for given function $w(x)$ the supremum of $W(n)$ for $n \in Z$. In view of Theorem 1 and its proof we can first find, using for example approximation methods, the only zero $n_{0} \in \mathbf{R}$ of the expression (8), and then look at which one of two values $W\left(\left[n_{0}\right]\right)$ or $W\left(\left[n_{0}\right]+1\right)$ is greater.

REMARK 2. In the case $w(x) \equiv 1$ it is easy to verify that $W^{\prime}(-1)=0$; thus $n_{0}=-1$ does not depend on $a, b, c, d$ (see [1]). More generally if $w(x)=x^{k}, k \in \mathbf{R}$ then $n_{0}=-k-1$ and this also does not depend on $a, b, c, d$. In the particularly interesting case of the Fock space, that is, $w(x)=\exp (-x)$, the situation is not so easy and one can show that $n_{0}$ does depend on $a, b, c, d$. 


\section{Continuity of The $L^{2}$-ANgle for the Fock SPaCe}

Let $A_{0}=\left\{z \in C:|z|<r_{2}\right\}$ and $B_{\infty}=\left\{z \in C:|z|>R_{1}\right\} . A$ and $B$ are the same as previously and $r_{1}<R_{1}<r_{2}<R_{2}, a=r_{1}^{2}, b=R_{1}^{2}, c=r_{2}^{2}, d=R_{2}^{2}$, $w(x)=\exp (-x)$.

A similar calculation as in [1] that we omit here yields:

$$
\cos ^{2} \gamma\left(A_{0}, B\right)=\sup _{n \in Z} U(n)
$$

where

$$
\begin{aligned}
U(n) & =\frac{\int_{c}^{d} \exp (-x) x^{n} d x}{\int_{b}^{d} \exp (-x) x^{n} d x} \text { for } n \leqslant-1 \\
U(n) & =\frac{\int_{0}^{b} \exp (-x) x^{n} d x \cdot \int_{c}^{d} \exp (-x) x^{n} d x}{\int_{0}^{c} \exp (-x) x^{n} d x \cdot \int_{b}^{d} \exp (-x) x^{n} d x} \quad \text { for } n \geqslant 0, \\
\cos ^{2} \gamma\left(A, B_{\infty}\right) & =\sup _{n \in Z} V(n) \\
V(n) & =\frac{\int_{a}^{b} \exp (-x) x^{n} d x \cdot \int_{c}^{\infty} \exp (-x) x^{n} d x}{\int_{a}^{c} \exp (-x) x^{n} d x \cdot \int_{b}^{\infty} \exp (-x) x^{n} d x}
\end{aligned}
$$

where

and finally

$$
\cos ^{2} \gamma\left(A_{0}, B_{\infty}\right)=\sup _{n \in Z} T(n)
$$

where $T(n)=\lim _{d \rightarrow \infty} U(n)$.

It is easy to see that for $n \leqslant-1$,

$$
\lim _{a \rightarrow 0^{+}} \frac{\int_{a}^{b} \exp (-x) x^{n} d x}{\int_{a}^{c} \exp (-x) x^{n} d x}=1
$$

By Lemma 2 and (20) we have for any $n \in Z$

$$
\begin{aligned}
& W(n)<U(n)<T(n) \\
& W(n)<V(n)<T(n)
\end{aligned}
$$

and moreover

$$
\begin{array}{ll}
\lim _{a \rightarrow 0^{+}} W(n)=U(n), & \lim _{d \rightarrow \infty} U(n)=T(n), \\
\lim _{d \rightarrow \infty} W(n)=V(n), & \lim _{a \rightarrow 0^{+}} V(n)=T(n) .
\end{array}
$$


By (21), (22), and because the functions $W(n), U(n), V(n), T(n)$ are bounded, we obtain successively

$$
\begin{aligned}
\lim _{a \rightarrow 0^{+}} \sup _{n \in Z} W(n) & =\sup _{n \in Z} U(n), \\
\lim _{d \rightarrow \infty} \sup _{n \in Z} W(n) & =\sup _{n \in Z} V(n), \\
\lim _{d \rightarrow \infty} \sup _{n \in Z} U(n) & =\lim _{a \rightarrow 0^{+}} \sup _{n \in Z} V(n)=\sup _{n \in Z} T(n) .
\end{aligned}
$$

In other words (23) means that (in contrast to [1]) the $L^{2}$-angle between rings for the Fock space is continuous.

REMARK 3. The discontinuity of the $L^{2}$-angle in case of the uniform weight (see [1], Theorem 3) is caused by the fact that the function $z^{-1}$ is neither in $L^{2} H\left(A_{0} \backslash\{0\}\right)$ nor in $L^{2} H\left(B_{\infty}\right)$.

\section{REFERENCES}

[1] J. Jakóbczak and T. Mazur, 'On discontinuity of $L^{2}$-angle', J. Austral. Math. Soc. 47 (1989), 269-279.

[2] I.P. Ramadanov and M.L. Skwarczyński, 'An angle in $L^{2}(C)$ determined by two plane domains', Bull. Polish Acad. Sci. Math. 32 (1984), 653-659.

[3] M. Skwarczyński, 'Alternating projections in complex analysis', in Complex Analysis and Applications, Editors L. Miev and I. Ramadanov, pp. 192-199 (Bulgar. Acad. Sci., 1985).

[4] M. Skwarczyński, 'A general description of the Bergman projection', Ann. Polon. Math. 46 (1985), 311-315.

[5] M. Skwarczyński, 'Alternating projections between a strip and a halfplane', Math. Proc. Cambridge Philos. Soc. 102 (1987), 121-129.

[6] M. Skwarczyński, ' $L^{2}$-angles between one-dimensional tubes', Studia Math. 90 (1988), 213-233.

Institute of Mathematics

Agricultural University

Nowoursynowska 166

02-766 Warsaw

Poland 